\title{
The Design and Application of Paperless Examination System
}

\author{
Amei Zhang \\ Xi’an International University, Shaanxi Xi’an 710077, China
}

Keywords: Paperless; examination system; design; application.

\begin{abstract}
In this new era that is equipped with high network technology, the design and application of paperless examination system which utilizes the network technology has become a reality. This paper will introduce the design and application process of paperless examination system in detail, in order to help people master and use this system better. The paperless examination system which is described in this paper is built on the current mature and well-developed Java technology. Java has become the common programming technology for intelligent network technology at present, the use of which can improve the compatibility and usability of the paperless test system effectively, so that it can facilitate the application effect.
\end{abstract}

\section{Introduction}

The rapid development of network information technology and the gradual popularization of the concept of environmental protection are the root causes for the success use of the paperless examination system. In the traditional examinations, the production of a piece of paper needs a lot of manpower, material resources and time resources, such as the process of setting questions, files making, printing, examining, marking, scoring, analyzing and so on. Paperless exams can simplify the examination process and reduce the consumption of teacher's energy and time, so that teachers can have more time and energy to improve the quality of teaching, rather than the examination process. Based on the perfect information technology, paperless examination does not need to present on paper. Therefore, it increases the flexibility and systematic of the examination, so that teachers can design the test content better according to their own needs. Because paperless exams do not require the use of paper-like resources, trees and water are greatly conserved.

\section{The design of the paperless examination system}

The functional requirements of paperless examination system are divided into paper production, the selection of the form of test contents, examination organization, examination process, evaluating results, management and so on. In order to meet the requirements of different test contents, paperless examination system should have a mass, scientific and objective test database, so that teachers can produce the papers based on the ability of students and the examination requirements, having the clear thoughts of the knowledge points and difficulties. Besides, the question bank must be updated regularly, which can consists with the students' newest textbook every year, and delete the questions that do not meet the requirements of the course or the outdated ones while adding more updated topics.

However, the paperless examination system is just another form and method of examination in all. Therefore, the merits of the traditional examinations should be retained. In traditional examinations, the form of examination questions is divided into two parts, namely objective questions and application questions. The objective questions are often the multiple-choice and checking questions which assess student's mastery of the basic knowledge. Application questions are designed to mainly check the student's capabilities of application, design and development of knowledge. Paperless examination system should choose these two types of questions reasonably, and allow the students to complete within the required time.

Traditional examinations are organized by the teachers or the educational administration department, and the examination process is supervised in order to prevent cheating. However, the 
paperless examination system must ensure that the display of examination notes before the test, and the candidates will not affect each other during the examination, and the papers will not be attacked or leaked by the LAN or software after the examination.

After the beginning of the examination, paperless examination system should supervise the whole examination process. If the students do not complete within the required time, the system will hand the paper automatically. If the computer happens to crash, restart or so on during the examination, the answers must be ensured to be saved before. When student completes the exam, the system can generate his scores automatically, and generate the Excel form sending to teachers and academic management system automatically. In addition, the paperless examination system also needs to have management capabilities, high security, versatility and good scalability, so that teachers and educational administration can design the examination content and manage the examination well, ensuring the quality of the examination.

The paperless examination system should have the following functions: (1) student information management: student information query, editing, import and export; (2) test database management: test information management, test import and export; (3) test information management: the test setting, test generation; (4) evaluation management: manual scoring, papers analysis and database consolidation(5) common tools: test data consolidation.

In the student information management, we can search the relevant information according to the student's name and his number. Student's information which comes mainly from the system's database will be displayed in the form of tabular. Through the editing and the import and export process of the student information, we can complete the basic management of student information, such as the addition, deletion or modification of the basic information of students.

The realization of question bank information management is through the test information management and the test import and export. Teachers can choose the appropriate questions according to the requirements of the examination, and divide them into three levels according to the difficulty of the subject. At the same time, teachers set the examination time, examination notes and so on. As the questions are divided into application questions and objective questions, the system can not evaluate the scores by itself. Therefore, the manually evaluation of teachers are necessary in the application questions while the objective questions can be reviewed and analyzed by the computer system. After that, the system will display the scores in the form of a table. After importing the form, the teacher can archive or delete it as needed. The test data consolidation is mainly to help merge student's scores, and to display them in the service terminal.

\section{The application of paperless examination system}

When students use their own student number and password to successfully log into the examination system, the students will stay in the examination instructions interface. In the bottom right of the interface, there is a timer which is used to countdown, and the schedule time is 100 seconds. Students must read the instructions in 100 seconds and then click any button to enter the exam interface and start the exam. If 100 seconds countdown ends and students still do not enter the examination interface, the system will enter the examination interface automatically. In the test interface, the system will save the answer and time every minute, and sent them to the server terminal. During the examination time, students can click "Confirm" button to complete the examination after they finish the paper. If the students do not finish the examination within the schedule time, the system will finish the exam automatically. The students will not be permitted to hand the paper within the first 30 minutes after the commencement of the examination. Otherwise, the interface will issue the warning voluntarily.

In order to ensure the security of the paperless test system and reduce the impact of the accidents on the student examinations, such as power failure, computer crash and so on, we must consider the problem of maximum preservation of the students answer. After the system gets right and the input of student's information, the secondary login can restore the information preserved before the system crashes. In this way, we can restore the information of student's answers with the largest limit. During the design process of this system, it is impossible to fully preserve the student's 
answers. Because this requires the consistency of data updating between the client-side and the server terminal, in order to ensure the frequent data exchange between them. This will require a lot of data transmission in a short time, and thus will cause great pressure to the network and the server. And this kind of pressure will increase as the number of student clients increases. When it increases to a certain extent, it will contribute to the crash of network or system.

In the paperless examination system, the objective questions are often checked by the system automatically. Objective questions mainly consist of multiple-choice and true-false question, which have their own standard answers. In the establishment of the question bank of the system, we need to input the standard answer together with these objective questions. During the question selection process, we should enclose both the objective questions and their answers to the test. When the examination is over, the system will compare the standard answer with the student's answer automatically, and give the specific score according to the score of each question. Subjective questions are checked by teachers manually. When teachers finished, they should enter the scores to the system. Therefore, the scores of subjective and objective questions can be calculated by the system, and then the final results are entered into the server terminal. The final scores are organized by the server and sent to the teachers and the information terminal of academic departments in the form of Excel files.

The users of paperless examination system are mainly three types, that is, administrators, teachers and students. The system needs to encrypt the login password based on the characteristics and needs of different groups of people. MD5 encryption algorithm is a traditional encryption, which can compress the digital private password which includes a large amount of information into a confidential format. The ciphertext encrypted by MD5 is irreversible, thus ensuring the security of the login password effectively. When a user logs in the system, the system converts the password which is entered by the user into an MD5, and then compared it with the data file password in the system. If the password is correct, the users will log in successfully. Otherwise, the users will be prohibited to $\log$ in.

There are two main purposes of encrypting an examination file: to prevent the disclosure of examination contents; to prevent others modifying the test paper or the answer with "hacking technology". The system adopts the Hash Map data structure and DES encryption algorithm apart from the Java Beans serialization to encrypt this kind of file. DES is a standard encryption algorithm that is widely used in various secure and secret places. DES encryption algorithm changes the 64-bit plaintext into the combination of 56-bit key and 8-bit parity bit by changing the 16-bit encoding into 64-bit cipher text output block.

\section{Summary}

The application of the paperless examination system can improve the quality of examinations effectively, which can liberate teachers from the complicated examination preparation work, and reduce the consumption of natural resources. During the design and application process of the paperless examination system, the designer should take the advantages of the traditional examination, while, minimize the impacts of the accident happening on the examination process and the information that the students have answered. Besides, the designer should pay attention to the safety of the examination documents and landing passwords. With the network technology is becoming more and more mature and popular, paperless examination system will have a better development and popularization.

\section{References}

[1] Zhang yongheng, The Design and Implementation of Paperless Examination Online Examination System Based on SSH Architecture[J],Journal of Yulin College,2012,04:61-62+73.

[2] Liu yunping, The Design and Implementation of Paperless Examination System [J], Journal of Taiyuan Normal University, 2012,03:86-89. 
[3] Shi jianfan, Design and Implementation of Paperless Examination System in Colleges and Universitie[J]. Science \& Technology, 2013, 26:363+400.

[4] Long ying, Wu qinghua, Wang xinhui, Zhang zhen, Design and Application of Examination Management System for Paperless Examination [J], Science \& Technology Review, 2013, 28:10-11.

[5] Zhou chen, Lu zheng, Gao ying, Zha yanfang, Cheng long. Design and Implementation of Paperless Examination System Based on SSH [J], 2013, 12:103-106+113.

[6] Chen sihui, Design and Key Technology Research of Paperless Examination System Based on Network[J],Computer Knowledge and Technology,2011,05:989-991+993.

[7] Li ruixia,Li zhiwei, Research on the Necessity of Applying Paperless Examination System in Universities[J],Computer CD - ROM Software and Application,2015,03:216+218.

[8] Zhang liangmiao, zhang xiaoping, liu liang, Liu xu, Rao weiwei, Research and Application of Paperless Network Examination System [J], college education, 2014, 04:89-90. 